\title{
Exercise Acutely Improves Dynamic Balance in Individuals with Unilateral Knee Osteoarthritis
}

\author{
Emma C. Goddard, James P. Dickey* \\ School of Kinesiology, Western University, Canada
}

Copyright $\mathrm{C} 2019$ by authors, all rights reserved. Authors agree that this article remains permanently open access under the terms of the Creative Commons Attribution License 4.0 International License

\begin{abstract}
Exercise is often used to manage knee osteoarthritis (OA). However, OA leads to balance impairments which may lead to injury. The purpose of this study was to determine the effect of exercise on dynamic balance of individuals with unilateral knee OA. On one occasion, twenty participants with unilateral knee OA completed the star excursion balance test (SEBT) in all eight directions (anterior, anteromedial, medial, posteromedial, posterior, posterolateral, lateral, and anterolateral) on both affected and unaffected limbs, before and after a supervised exercise program. The exercise program consisted of 30 minutes of stretching and strengthening of the knee flexors and extensors. The SEBT was quantified as the composite score from all 8 directions. Participants also completed a Visual Analogue Scale (VAS) of pain before and after exercise. A two-way repeated-measures ANOVA revealed no significant interaction nor difference between affected and unaffected sides for SEBT. There was significant improvement in SEBT composite score after exercise $(7 \%$ and $5 \%$ post exercise for the affected and unaffected limbs respectively). VAS was not significantly different before and after exercise. These results suggest that a single bout of exercise improves acute dynamic balance performance in both affected and unaffected limbs in individuals with unilateral knee OA.
\end{abstract}

Keywords Biomechanics, Knee Osteoarthritis, Balance, Exercise, Star Excursion Balance Test (SEBT)

\section{Introduction}

Osteoarthritis (OA) is a non-inflammatory disorder of synovial joints that is characterized by loss of hyaline cartilage and remodeling of the surrounding bone [1]. The knee is one of the most commonly affected joints, and poses a significant burden to the healthcare system worldwide, affecting over 241 million people [2]. Knee OA results in significant pain, decreased quadriceps muscle strength, and impaired postural control and joint proprioception [3]. Individuals suffering from knee OA experience a progressive loss of function due to their symptoms [4]. These symptoms can also lead to balance impairments in individuals with OA compared to controls [5].

Exercise is an important component in managing OA, irrespective of severity, because it reduces pain and improves function [1]. Regular exercise, including stretching and strengthening (often in exercise and/or physiotherapy programs), is the standard of care for individuals with knee OA [6]. Additionally, exercise programs have been shown to improve balance when evaluated long term [7, 8]. However, there is a lack of research evaluating the acute effects of exercise in this population.

It has been reported that pre-exercise warm-ups improve the dynamic and static balance of healthy populations [9]. Therefore, exercise may acutely facilitate neuromuscular control, reduce stiffness and significantly improve dynamic balance in individuals with knee OA. Alternatively, muscular fatigue that may acutely negate these benefits [10].

Given that falls are a devastating injury in the elderly [11], and that prescribed exercise programs improve balance $[12,13]$, it is important to evaluate the acute effects of a single bout of exercise on balance performance in individuals with $\mathrm{OA}$.

The purpose of this study was to determine the acute effect of exercise on dynamic balance of individuals with knee OA. It was hypothesized that individuals with unilateral knee OA would have larger composite star excursion balance test (SEBT) reach distance after completing an exercise session. It was also hypothesized that the changes would be larger on the contralateral limb than on the limb with osteoarthritis.

\section{Materials and Methods}

\subsection{Study Design}

This study used an experimental, repeated measures 
design evaluating the effects of a single session of exercise on dynamic balance by measuring the Star Excursion Balance Test (SEBT) before and after an exercise session. Participants attended one experimental session.

\subsection{Participants}

Individuals with clinical knee $\mathrm{OA}$ according to the Altman classification were eligible for the study [14]. The Altman classification requires knee pain with at least three of six clinical findings including age greater than 50 years, morning stiffness less than 30 minutes, crepitus, bony tenderness on the joint, bony enlargement, and lack of palpable warmth [14]. Exclusion criteria included previous joint replacement, inflammatory or infectious arthritis of the knee, major neurological disorder, major medical illness, inability to read English, psychiatric illness that limits informed consent, inability to exercise for 30 minutes, and inability to stand on one limb for five seconds.

The study participants were recruited via posters at a community physician's office. A sample of 20 individuals with unilateral knee OA, 5 men and 15 women, aged $64 \pm 9$ years, were tested.

\subsection{Outcome Measures}

The star excursion balance test (SEBT) was performed using all eight directions [15]. Pain was assessed before and after exercise session using a Visual Analogue Scale (VAS). The Knee Injury and Osteoarthritis Outcome Score (KOOS) was assessed at beginning of the session. The participant's age, height, weight and leg length (anterior superior iliac spine to the ipsilateral medial malleolus) were recorded. Balance measurements were performed on both limbs, with the participants' contralateral limb used as a control.

\subsubsection{Visual Analogue Scale of Pain}

A VAS was used to measure pain before and after the exercise session, preceding the SEBT protocols. The VAS is a tool that measures a characteristic (pain) on a horizontal line continuum, $100 \mathrm{~mm}$ in length, with visual labels designating that the left side indicates no pain and the right side indicates most possible pain [16]. The VAS is a reliable tool for looking at change within an individual $[16,17]$.

\subsubsection{KOOS}

The KOOS is a 42-item self-administered questionnaire that covers five patient-relevant dimensions: pain (9 questions), other disease-specific symptoms (7 questions), daily living function (17 questions), sport and recreation function (5 questions), and knee-related quality of life (4 questions). The KOOS is the most commonly used outcome instrument for assessment of patient-relevant treatment effects in osteoarthritis, and it takes approximately 10 minutes to administer [18]. Each subscale has a number of questions that are rated with a Likert scale from zero to four. The total score ranges from $0-100$, where a score of zero indicates extreme knee problems while a score of 100 indicates no knee problems [18].

\subsubsection{SEBT}

There is considerable variability in the way that different studies have performed the SEBT protocol. This study was modeled after research that also evaluated individuals with knee OA [8]. The SEBT was performed using eight lines taped to the floor, each at $45^{\circ}$ to each other, with centimeters marked to determine reach distance (Figure 1). Participants performed the test barefoot. The participant positioned their stance leg at the centre of the star, with the first medial cuneiform and arch of the foot over the centre mark. The participant reached with the opposite leg as far as possible in the specified direction while maintaining balance on the stance leg. They made a light touch with their toe at the maximal reach and returned to the original double leg stance position. The average of the reach lengths in each of the eight directions was normalized as a percentage of leg length [19]. Leg length is defined as the length from the anterior superior iliac spine (ASIS) to the most distal portion of the medial malleolus [19]. The composite score was calculated as the average of the eight reach directions [20].

Right Stance Leg

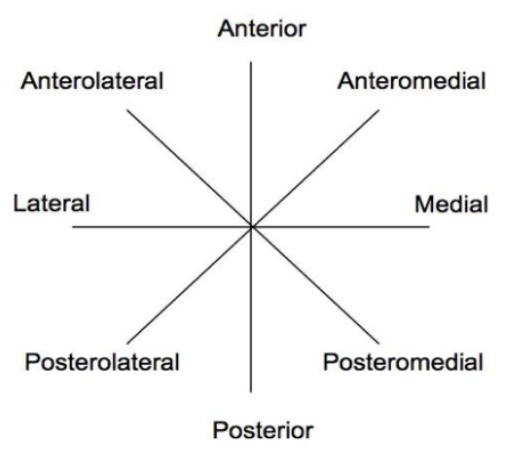

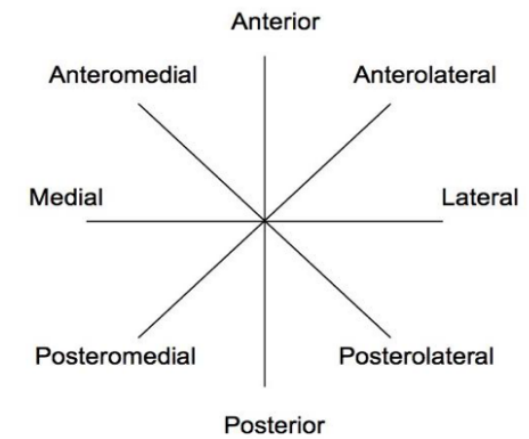

Figure 1. The star excursion balance test set up for the left and right stance legs. 
The participant was required to keep their hands on their hips for the entire trial, and the stance foot could not move. One observer performed all measurements to reduce variability. They monitored the participant's position and record the maximal reach distance for each trial. Trials were discarded and repeated if the observer determined that 1) an appropriate position of the stance limb was not maintained with the knee moving out of line with the toe, 2) the stance foot was lifted or moved from the centre of the grid, 3) the participant did not touch down, or touched down more than once, during the trial, 4) considerable support was put in the reaching leg when touching the ground, 5) the participant lost balance at any point or failed to return to the starting position, 6) their hands came off their hips.

All participants received verbal instructions and watched a demonstration of the test before completing the SEBT. One practice trial was performed standing on the unaffected leg in each of the eight directions, and one practice trial was repeated in each direction standing on the affected leg. The order of test direction was performed as follows, relative to stance leg: anterior, anteromedial, medial, posteromedial, posterior, posterolateral, lateral, and anterolateral (Figure 1). Three trials were recorded consecutively for each test direction and the average was calculated and used in subsequent analyses. All participants performed the SEBT on their unaffected leg first and then on their affected leg.

\subsection{Statistical Analysis}

The statistical significance of differences in composite reach scores, and VAS scores, from before and after the exercise session, and between the arthritic and non-arthritic limbs, were determined using two-way repeated measures ANOVAs. All statistical analyses were performed using GraphPad Prism (version 6.00 for Mac OS, GraphPad Software, La Jolla California USA, www.graphpad.com). Partial Eta squared values of effect size $\left(\eta_{\mathrm{p}}{ }^{2}\right)$ were calculated using an open-access spreadsheet (http://openscienceframework.org/project/ixGcd).

\subsection{Exercise Program}

This study used an established exercise routine that emphasizes optimal alignment of the trunk and lower limb joints relative to one another, as well as quality of movement performance, while dynamically and functionally strengthening the lower limb muscles [6]. It is fully described in the supplementary material associated with [6], and can be accessed at https://static-content.springer.com/esm/art\%3A $10.1186 \%$ 2F1471-2474-12-276/MediaObjects/12891_2011_1294 MOESM1_ESM.doc. All of the stretches and exercises were completed on both the affected and unaffected limb. The exercise program took approximately $30-40$ minutes to complete.

\section{Results}

The KOOS results indicated that all participants suffered from moderate to severe symptoms in their affected limb (Table 1). There was no statistically significant interaction for the VAS score between the affected/unaffected limb and pre/post exercise $\left(\mathrm{F}=0.24, \mathrm{p}=0.63, \eta_{\mathrm{p}}{ }^{2}=0.01\right.$; Figure 2). There was a significant main effect of limb on the VAS score between pre- and post-exercise conditions $(\mathrm{F}=36.27$, $\mathrm{p}<0.0001, \eta_{\mathrm{p}}{ }^{2}=0.66$; Figure 2), but no significant main effect of time ( $\mathrm{F}=0.13, \mathrm{p}=0.72, \eta_{\mathrm{p}}{ }^{2}=0.01$; Figure 2).

Table 1. KOOS score of subscales Pain, Symptoms, Activities of Daily Living, Sport and Recreation, and Quality of Life.

\begin{tabular}{|c|c|}
\hline Subscale* $^{*}$ & mean $+/-$ SD \\
\hline Pain & $53.70+/-15.42$ \\
\hline Symptoms & $52.55+/-15.12$ \\
\hline ADL & $60.69+/-17.51$ \\
\hline Sport/Rec & $33.10+/-19.40$ \\
\hline QOL & $41.37+/-18.59$ \\
\hline
\end{tabular}

*A score of 100 indicates no knee problems and 0 indicates extreme knee problems.

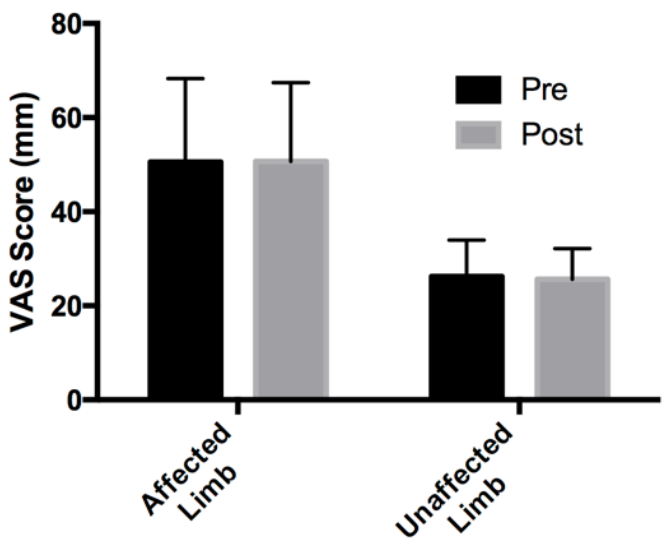

Figure 2. Mean VAS score on a $100 \mathrm{~mm}$ continuum from before and after exercise on both the affected and unaffected limbs. There was a statistically significant difference between limbs, but no significant interaction and no significant difference between pre and post exercise.

Considering the composite reach scores, there was no statistically significant interaction between the affected/unaffected limb and pre/post exercise $(\mathrm{F}=2.1$, $\mathrm{p}=0.16, \eta_{\mathrm{p}}{ }^{2}=0.12$; Figure 3 ). There was a significant main effect of time on composite reach score between pre- and post-exercise conditions $\left(\mathrm{F}=83.43, \mathrm{p}<0.0001, \eta_{\mathrm{p}}{ }^{2}=0.77\right.$; Figure 3).

The mean composite reach score improved $7 \%$ and $5 \%$ post exercise for both the affected and unaffected limbs, respectively. There was no significant main effect of affected/unaffected limb ( $\left.\mathrm{F}=0.05, \mathrm{p}=0.83, \eta_{\mathrm{p}}{ }^{2}=0.04\right)$. 


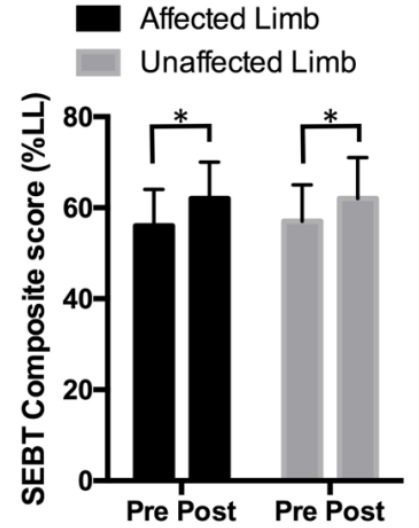

* Statistically significant mean difference $(p<0.05)$

Figure 3. Mean normalized (\% of leg length) composite SEBT reach values before and after exercise for both the affected and unaffected legs.

\section{Discussion}

The purpose of this study was to determine the acute effect of exercise on dynamic balance of individuals with unilateral knee osteoarthritis. It was hypothesized that individuals with knee OA would have a larger composite SEBT reach distance after completing an exercise session. We observed a significant increase in balance performance after the exercise session on both the affected and unaffected leg, which is consistent with our hypothesis. The medium effect size indicates that this increase has practical significance. Additionally, the VAS data also suggests that a single exercise session does not acutely increase pain in limbs with knee OA.

It was also hypothesized that the changes would be larger on the contralateral limb than on the limb with osteoarthritis. There was no significant difference between affected and unaffected limbs either before or after exercise, and no significant interaction, which does not support this hypothesis. There was a trivial effect size for all of these comparisons supporting that they are not practically important. There are several potential factors that could have contributed to this observation. For example, recent research evaluated three SEBT reach directions (y-balance test) and determined that the posteromedial and posterolateral reach directions of the SEBT can detect neuromuscular control deficits in individuals with anterior cruciate ligament deficiency, but that the anterior reach direction was not different [21]. In contrast, other researchers have reported significant differences in the anterior reach distances in individuals with lateral ankle sprains compared to controls [22]. Accordingly, our quantification of the SEBT using the composite reach measure, which incorporates all eight reach directions, may effectively dilute changes in individual reach directions.

Electromyography (EMG) data has suggested that gastrocnemius, gluteus maximus, and vastus medialis were activated for every direction of the SEBT, but that the remainder of the hip/thigh and lower extremity muscle activation in healthy populations varied between reach directions [23, 24]. Studies focusing on muscle activation during gait in individuals with knee OA have concluded that muscle activation patterns change with increased severity of OA [25]. Additionally, it has been reported that during both level and stair climbing ambulation, patients with knee OA were able to complete the tasks with the same limb acceleration and movement compared to a control group despite EMG data demonstrating differentiated muscle group activation and coordination [26]. It is possible that the SEBT could be performed using compensatory muscle activation patterns, therefore reducing reach distance differentiation between limbs. Muscle activation was not monitored during the present study; therefore it is unclear if the same muscle activation patterns were present, which could have affected the outcome. Additionally, joint kinematics were not observed. When the SEBT has been used in injured populations, often kinematic differences are evident between affected and control tests, particularly in hip and knee flexion [22]. Joint kinematic and muscle activation data would be helpful in order to evaluate whether the SEBT movements were performed the same way in each test.

If joint impairments are only a local result of knee OA, then we would expect to observe a difference between limbs in individuals with unilateral knee OA. One investigation evaluating patients with lateral ankle sprains demonstrated that joint flexion was different at both the hip and knee on both the affected and unaffected limb during the SEBT [22]. Despite the unilateral injury, there was a clear bilateral impairment compared to healthy controls [22]. A similar bilateral effect was observed as individuals with unilateral knee OA have reduced joint proprioception in their contralateral limb and their affected limb [27]. This suggests that the poor spatial and temporal coordination that affects kinematics caused by knee OA is not necessarily localized to the affected limb [27]. It could be the case in the present study that the OA impairment has affected both limbs. This might account for the lack of between-limb difference. If unilateral $\mathrm{OA}$ is impacting function of both limbs, it is a further indication that exercise should be included in clinical management as it would be beneficial for both limbs.

The increase in SEBT composite score in the both the affected and unaffected limb observed in the present study is greater than the change observed in individuals with OA following a 12 week exercise program $(4.46 \%$ increase in balance performance on the affected limb) [8]. Exercise treatment in individuals with OA has been shown to reduce pain, improve strength, and improve function [1, 7]. This has been shown help reduce risk of falls in this population [7]. The KOOS data indicated that all participants in the present study experienced symptoms ranging from moderate to severe that affected their daily activities and quality of life. Previous studies have 
reported that the observed improvements in balance with a six-week exercise program were comparable to the results of the present study [28]. These balance improvements were accompanied by improved KOOS scores in pain and activities of daily living [28]. This suggests that the observed increase in dynamic balance performance in the present study may be functionally important for individuals suffering from $\mathrm{OA}$.

Some research has suggested that neuromuscular fatigue may negatively affect balance and postural control acutely $[10,29]$, but the present study did not observe any negative changes in performance due to fatigue. The exercises utilized in the present study were clinician approved and consistent with guidelines for treatment of knee OA $[1,6]$. The balance improvements observed in the present study suggest that exercise has an acute benefit for individuals with knee $\mathrm{OA}$, in addition to the previously established long-term benefits.

\subsection{Limitations}

A potential limitation of this study is the lack of control for diurnal variation. Knee OA symptoms, such as joint pain and stiffness, fluctuate throughout the day in the OA patients [30]. Clinical symptoms have been shown to effect dynamic balance and postural control, especially due to stiffness in the morning [31]. Therefore, the participants that were seen in the morning may have demonstrated more improvement than the people in early afternoon or evening, as they would not have had the same amount of stiffness [31].

Three quarters of the participants in this study were female. There are sex differences in the clinical presentation of knee OA due to knee anatomy, kinematics, and hormonal influence [32]. Accordingly our findings may not be generalizable, and should be interpreted with caution. We recommend that this study be repeated with a larger sample size, and a more equal number of male and female participants. This would provide evidence about the generalizability of our findings.

Previous literature supports the use of four practice trials and three test trials in the SEBT protocol in healthy individuals to account for learning effect [29, 33]. However, the present study evaluated all 8 directions in a population with knee $\mathrm{OA}$, which could be a significant physical burden. Therefore, in order to ensure that the participants could complete the test, the protocol was modified to one practice trial and three test trials. The average of three test trials was used to better represent individuals' true scores [34]. Therefore, there is the potential that a learning effect occurred in the present study.

\section{Conclusions}

In conclusion, these results suggest that a single exercise session does not acutely increase pain in limbs with knee OA. The results also suggest that exercise improves dynamic balance performance in both affected and unaffected limbs in individuals with unilateral knee OA. This improved dynamic balance could potentially increase limb function for affected populations. The absence of pain or reduced dynamic balance ability suggests that there are no contraindications to population specific exercise for individuals with unilateral knee OA. This supports the clinical use of exercise for the management of unilateral knee OA.

\section{Acknowledgements}

This project was completed as part of the course requirements for Kinesiology 4443, Research Project in Kinesiology, at the University of Western Ontario. We would like to extend thanks to all of the participants that volunteered to be a part of this study. We would also like to thank Ellen Ibey and the rest of the Temagami Family Health Team for their gracious cooperation with recruitment efforts and data collection.

\section{REFERENCES}

[1] Walker J. (2011). Management of osteoarthritis. Nursing Older People. 23(9):14-19. doi: 10.7748/nop2011.11.23.9.1 4.c8778

[2] Cross M., Smith E., Hoy D., Nolte S., Ackerman I., Fransen M., et al. (2014). The global burden of hip and knee osteoarthritis: estimates from the global burden of disease 2010 study. Annals of the Rheumatic Diseases. 73(7):1323-1330. doi: 10.1136/annrheumdis-2013-204763

[3] Hurley M.V., Scott D.L., Rees J., Newham D.J. (1997). Sensorimotor changes and functional performance in patients with knee osteoarthritis. Annals of the Rheumatic Diseases. 56(11):641-648. doi: 10.1136/ard.56.11.641

[4] Hinman R.S., Bennell K.L., Metcalf B.R., Crossley K.M. (2002). Balance impairments in individuals with symptomatic knee osteoarthritis: a comparison with matched controls using clinical tests. Rheumatology (Oxford, England). 41(12):1388-1394. doi: 10.1093/rheumatology/4 1.12 .1388

[5] Wegener L., Kisner C., Nichols D. (1997). Static and dynamic balance responses in persons with bilateral knee osteoarthritis. Journal of Orthopaedic and Sports Physical Therapy. 25(1):13-18. doi: 10.2519/jospt.1997.25.1.13

[6] Bennell K.L., Egerton T., Wrigley T.V., Hodges P.W., Hunt M., Roos E.M., et al. (2011). Comparison of neuromuscular and quadriceps strengthening exercise in the treatment of varus malaligned knees with medial knee osteoarthritis: a randomised controlled trial protocol. BMC Musculoskeletal Disorders. 12:276. doi: 10.1186/1471-2474-12-276

[7] Mat S., Tan M.P., Kamaruzzaman S.B., Ng C.T. (2015). 
Physical therapies for improving balance and reducing falls risk in osteoarthritis of the knee: a systematic review. Age and Ageing. 44(1):16-24. doi: 10.1093/ageing/afu112

[8] Kanko L.E., Birmingham T.B., Bryant D.M., Gillanders K., Lemmon K., Chan R., et al. (2018). The star excursion balance test is a reliable and valid outcome measure for patients with knee osteoarthritis. Osteoarthritis and Cartilage. in press. doi: 10.1016/j.joca.2018.11.012

[9] Daneshjoo A., Mokhtar A.H., Rahnama N., Yusof A. (2012). The effects of comprehensive warm-up programs on proprioception, static and dynamic balance on male soccer players. PloS One. 7(12):e51568. doi: 10.1371/journal.pone.0051568

[10] Nardone A., Tarantola J., Giordano A., Schieppati M. (1997). Fatigue effects on body balance. ElectroencephalogrClin Neurophysiol. 105(4):309-320. doi: 10.1016/S0924-980X(9 7)00040-4

[11] Sri-On J., Tirrell G.P., Bean J.F., Lipsitz L.A., Liu S.W. (2017). Revisit, Subsequent Hospitalization, Recurrent Fall, and Death Within 6 Months After a Fall Among Elderly Emergency Department Patients. Annals of Emergency Medicine. 70(4):516-521 e512. doi: 10.1016/j.annemergme d.2017.05.023

[12] Carter N.D., Kannus P., Khan K.M. (2001). Exercise in the prevention of falls in older people: a systematic literature review examining the rationale and the evidence. Sports Medicine. 31(6):427-438. doi: 10.2165/00007256-2001310 60-00003

[13] Tse A.C., Wong T.W., Lee P.H. (2015). Effect of Low-intensity Exercise on Physical and Cognitive Health in Older Adults: a Systematic Review. Sports Med Open. 1(1):37. doi: 10.1186/s40798-015-0034-8

[14] Altman R., Asch E., Bloch D., Bole G., Borenstein D., Brandt K., et al. (1986). Development of criteria for the classification and reporting of osteoarthritis: Classification of osteoarthritis of the knee. Arthritis and Rheumatism. 29(8):1039-1049. doi: 10.1002/art.1780290816

[15] Hertel J., Miller S.J., Denegar C.R. (2000). Intratester and intertester reliability during the Star Excursion Balance Tests. Journal of sport rehabilitation. 9:104-116. doi: 10.1123/jsr.9.2.104

[16] Gould D., Kelly D., Goldstone L., Gammon J. (2001). Examining the validity of pressure ulcer risk assessment scales: developing and using illustrated patient simulations to collect the data. Journal of Clinical Nursing. 10(5):697-706. doi: 10.1046/j.1365-2702.2001.00525.x

[17] Bijur P.E., Silver W., Gallagher E.J. (2001). Reliability of the visual analog scale for measurement of acute pain. Academic Emergency Medicine. 8(12):1153-1157. doi: 10.1111/j.1553-2712.2001.tb01132.x

[18] Roos E.M., Toksvig-Larsen S. (2003). Knee injury and Osteoarthritis Outcome Score (KOOS) - validation and comparison to the WOMAC in total knee replacement. Health Qual Life Outcomes. 1:17. doi: $10.1186 / 1477-7525-1-17$

[19] Ayala F., Calderon-Lopez A., Delgado-Gosalbez J.C., Parra-Sanchez S., Pomares-Noguera C., Hernandez-Sanchez S., et al. (2017). Acute Effects of Three Neuromuscular
Warm-Up Strategies on Several Physical Performance Measures in Football Players. PloS One. 12(1):e0169660. doi: 10.1371/journal.pone.0169660

[20] Filipa A., Byrnes R., Paterno M.V., Myer G.D., Hewett T.E. (2010). Neuromuscular training improves performance on the star excursion balance test in young female athletes. Journal of Orthopaedic and Sports Physical Therapy. 40(9):551-558. doi: 10.2519/jospt.2010.3325

[21] Dobija L., Reynaud V., Pereira B., Van Hille W., Descamps S., Bonnin A., et al. (2018). Measurement properties of the Star Excursion Balance Test in patients with ACL deficiency. Physical Therapy in Sport 36:7-13. doi: 10.1016/j.ptsp.2018.12.010

[22] Doherty C., Bleakley C.M., Hertel J., Caulfield B., Ryan J., Delahunt E. (2015). Laboratory Measures of Postural Control During the Star Excursion Balance Test After Acute First-Time Lateral Ankle Sprain. $J$ Athl Train. 50(6):651-664. doi: 10.4085/1062-6050-50.1.09

[23] Earl J.E., Hertel J. (2001). Lower-extremity muscle activation during the Star Excursion Balance Tests. Journal of sport rehabilitation. 10(2):93-104. doi: DOI $10.1123 /$ jsr.10.2.93

[24] Norris B., Trudelle-Jackson E. (2011). Hip-and thigh-muscle activation during the star excursion balance test. Journal of sport rehabilitation. 20(4):428-441. doi: 10.1123 /jsr.20.4.428

[25] Rutherford D.J., Hubley-Kozey C.L., Stanish W.D. (2013). Changes in knee joint muscle activation patterns during walking associated with increased structural severity in knee osteoarthritis. Journal of Electromyography and Kinesiology. 23(3):704-711. doi: 10.1016/j.jelekin.2013.01. 003

[26] Liikavainio T., Bragge T., Hakkarainen M., Karjalainen P.A., Arokoski J.P. (2010). Gait and muscle activation changes in men with knee osteoarthritis. Knee. 17(1):69-76. doi: 10.1016/j.knee.2009.05.003

[27] Sharma L., Pai Y.C., Holtkamp K., Rymer W.Z. (1997). Is knee joint proprioception worse in the arthritic knee versus the unaffected knee in unilateral knee osteoarthritis? Arthritis and Rheumatism. 40(8):1518-1525. doi: 10.1002/art.1780400821

[28] Al-Khlaifat L., Herrington L.C., Tyson S.F., Hammond A., Jones R.K. (2016). The effectiveness of an exercise programme on dynamic balance in patients with medial knee osteoarthritis: A pilot study. The Knee. 23(5):849-856. doi: 10.1016/j.knee.2016.05.006

[29] Gribble P.A., Hertel J., Plisky P. (2012). Using the Star Excursion Balance Test to assess dynamic postural-control deficits and outcomes in lower extremity injury: a literature and systematic review. Journal of Athletic Training. 47:339-357. doi: 10.4085/1062-6050-47.3.08

[30] Bellamy N., Sothern R., Campbell J. (1990). Rhythmic variations in pain perception in osteoarthritis of the knee. The Journal of rheumatology. 17(3):364-372.

[31] Zhang Z., Lion A., Chary-Valckenaere I., Loeuille D., Rat A.C., Paysant J., et al. (2015). Diurnal variation on balance control in patients with symptomatic knee osteoarthritis. Archives of Gerontology and Geriatrics. 61(1):109-114. doi: 
10.1016/j.archger.2015.03.009

[32] Hame S.L., Alexander R.A. (2013). Knee osteoarthritis in women. Current Reviews in Musculoskeletal Medicine. 6(2):182-187. doi: 10.1007/s12178-013-9164-0

[33] Robinson R.H., Gribble P.A. (2008). Support for a reduction in the number of trials needed for the star excursion balance test. Archives of Physical Medicine and Rehabilitation. 89:364-370. doi: 10.1016/j.apmr.2007.08.139

[34] Gribble P.A., Hertel J. (2003). Considerations for Normalizing Measures of the Star Excursion Balance Test. Measurement in Physical Education and Exercise Science. 7:89-100. doi: 10.1207/S15327841MPEE0702_3 